\title{
Y papá lee el periódico. La perspectiva de género en la audiencia de los medios de comunicación y reflexiones de
} derecho antidiscriminatorio

And dad reads the newspaper. The gender perspective in media audiences and reflections on anti-discrimination law

Belén Trigo-García, Universidad de Santiago de Compostela - belen.trigo@usc.es

Abstract

Traditionally, the reading of a newspaper was considered a male practice. The reasons usually referred to the contents, not suitable or outside the sphere of female interests (v. gr., politics or business). Therefore, women often accessed to information through secondary and informal sources (family, friends). In short, it was not promoted that women formed their own opinion on key issues of public life. This contribution aims to analyze if a change can be observed in the audience of the media; if we can speak of diversity and pluralism or if gender stereotypes are still present.

Keywords

Communication, gender, diversity, anti-discrimination law.

Resumen

Tradicionalmente, la lectura de los diarios era considerada una práctica típicamente masculina. Las razones remitían, de forma usual, a los contenidos, no adecuados o ajenos a la esfera de intereses femeninos (v. gr., política o economía). Por tanto, el acceso de las mujeres a la información tenía lugar, con frecuencia, a través de fuentes secundarias e informales (familiares, amigos). En definitiva, no se promovía que la mujer formase una opinión propia sobre temas clave de la vida pública. La contribución pretende analizar hasta qué punto se ha producido un cambio cultural en la audiencia de los medios de comunicación; si puede hablarse de diversidad y pluralismo 0 si los estereotipos de género siguen presentes.

Palabras clave

Comunicación, género, diversidad, derecho antidiscriminatorio.

Sumario

1. Los medios de comunicación, recreación cultural y perspectiva de género. 2. Audiencia de los medios de comunicación según medios. 3. La audiencia de los medios de comunicación, género y contexto socioeconómico. Discriminación múltiple de la mujer. 4. Género, medición de audiencias y acceso a la información. 5. Bibliografía. 


\section{Los medios de comunicación, recreación cultural y perspectiva de género}

Los medios de comunicación contribuyen de forma indudable a la creación cultural; al mismo tiempo, recrean la cultura contemporánea. Esta recreación, si bien muestra la diversidad cultural en un momento dado, puede que no ofrezca un reflejo fiel de la misma al estar condicionada su representación por intereses económicos y comerciales, presupuestos ideológicos, y estereotipos sociales. En este sentido, los medios de comunicación pueden ser un poderoso instrumento de deformación de la realidad social (García de León, 2002: 8687).

El cotejo de los datos españoles sobre audiencia de los medios desglosados por sexo, combinado con el seguimiento de la evolución en los últimos años, ofrece una información útil para reflexionar sobre cómo los roles de género persisten en la sociedad actual, también en el ámbito de los medios de comunicación.

De entrada debe aclararse que se habla exclusivamente de diferencias culturales; por tanto, la referencia ha de ser propiamente al género, no al sexo'.

Por otra parte, resulta posible y esclarecedor completar la imagen que estos datos ofrecen con otros relacionados. En particular, se revisarán los resultados, también desglosados por sexos, relativos a la educación, el uso de tecnología, el tiempo de ocio, la dedicación a las tareas del hogar, y la empleabilidad.

En efecto, los medios de comunicación no son el único ámbito de creación y recreación cultural. Por otra parte, en el consumo de estos medios influyen factores como el nivel económico y formativo, el entorno social y familiar, así como las pautas de ocio y uso del tiempo libre.

Obviamente, se trata de una visión incompleta. Quedan fuera los resultados de las investigaciones llevadas a cabo en materia de visibilidad $^{2}$ y tratamiento de la mujer en los medios de comunicación en general y, en particular, de su papel en la publicidad y la mercadotecnia ${ }^{3}$.

Así lo reconoce de manera expresa la Ley Orgánica 3/2007, de 22 de marzo, para la igualdad efectiva de mujeres y hombres, cuyo Título III Igualdad y medios de comunicación, contiene medidas de fomento de la igualdad en los medios de comunicación social, con reglas específicas para los de titularidad pública4 ${ }^{4}$ La misma norma, contempla también la actividad publicitaria al hilo de la regulación de los medios de comunicación, considerando publicidad ilícita -con remisión a la legislación general de publicidad y de publicidad y comunicación institucional- la publicidad que comporte una conducta discriminatoria (art. 41 lgualdad y publicidad) (Macías, 2013: 207226).

Ahora bien, estos campos han sido ya tratados con cierta extensión y profundidad por lo que me centraré en los aspectos antes indicados. Y una última aclaración sobre el enfoque y objetivos de la presente contribución. Ciertamente, como antes se apuntó, la perspectiva prevalente es la de género; no obstante, entiendo que aun reconociendo la singularidad de esta línea de investigación, resulta enriquecedor contextualizarla dentro de la orientación más amplia del derecho antidiscriminatorio.

En efecto, como se explicará a continuación, no solo los estudios de género comparten la filosofía común del derecho antidiscriminatorio (López Díez, 2005: 67-90); además, en la práctica es frecuente la discriminación múltiple, esto es, la concurrencia en una misma persona de dos o más causas de discriminación. Situación fáctica que, entiendo, debe ser reconocida por el análisis teórico y académico.

\section{Audiencia de los medios de comunicación según medios}

El Instituto de la mujer y para la igualdad de oportunidades ofrece en el apartado «Ciencia y Tecnología» el resumen de los datos del Estudio General de Medios elaborado por la Asociación Internacional de Medios de comunicación entre los años 2002 y 2014 (datos actualizados a 24 de julio de 2014$)^{5}$.

La muestra recoge las preferencias del grupo de población a partir de 14 años cumplidos. Las opciones tienen en cuenta la tecnología y soporte del medio de comunicación, así como su periodicidad; se distingue entonces entre diarios, radio y televisión, respecto de los que se

\footnotetext{
1 Vid. la definición de Gender ofrecida por la OMS y su diferencia respecto de Sex (What do we mean by «sex» and «gender»?, disponible en http://www.who.int/gender/whatisgender/en/). Cfr. el glosario elaborado por la UNESCO (p. 53 del documento Gender-Sensitive Indicators for Media- Framework of Indicators to Gauge Gender Sensitivity in Media Operations and Content) disponible en http://unesdoc.unesco.org/images/0021/002178/217831e.pdf

2 Sobre la utilización del lenguaje, vid. Ignacio Bosque, "Sexismo lingüístico y visibilidad de la mujer", http://cultura.elpais.com/cultura/2012/03/02/actualidad/1330717685_771121.html.

3 Sobre la igualdad entre mujeres y hombres que trabajan en los medios, y la igualdad en la cobertura de las noticias, vid. Global Report on the Status of Women in the News Media 2011, disponible en http://www.iwmf.org/. Sin ánimo exhaustivo, Núñez Zorrilla, 2011: 921-955, Pérez Marín, 2011: 983-115, Rodríguez González, 2011: pp. 1017-1043, Martín García, 2011: 903-919, Berlanga Fernández, 2011: 145-160; Bernad Monferrer, 2012: 151-159. Ha de llamarse la atención sobre las repercusiones de la imagen de la mujer ofrecida por los medios de comunicación (Menéndez Menéndez, 2013:253-270; López Lita /Bernad Monferrer, 2007: 213-226).

${ }^{4}$ Art. 36 La igualdad en los medios de comunicación social de titularidad pública, art. 37 Corporación RTVE, y art. 38 Agencia EFE. En el caso de los medios privados, la ley remite a los acuerdos de autorregulación que aprueben (art. 39 La igualdad en los medios de comunicación social de titularidad privada).

${ }^{5}$ Disponible en http://www.inmujer.gob.es/estadisticas/consulta.do?area=4.
} 
contabiliza el consumo diario; y suplementos y cine, en los que las cifras se refieren a audiencia semanal. Respecto de las revistas, se consideran los lectores por el periodo de publicación.

Por lo que se refiere a internet, hasta 2007 los datos recogen el porcentaje de usuarios en el día anterior; mientras que a partir de ese año, reflejan el porcentaje de usuarios por mes. Dada esta diferencia de criterio, se atenderá preferentemente a los datos del periodo 2008-2014 respecto del uso de internet.

Los medios en los que prevalece la audiencia femenina son los suplementos, las revistas, la televisión y el cine; los medios en los que prevalece la audiencia masculina son los diarios, la radio e internet.

Las diferencias más significativas por sexos afectan a la lectura de diarios, suplementos y revistas; mientras que la evolución más acusada se ha producido respecto del uso de internet. El porcentaje de usuarias y usuarios de televisión, así como la penetración de este medio, se ha mantenido estable a lo largo del periodo, con escasas oscilaciones y un reparto prácticamente igualitario por sexos.

De forma parecida, el reparto de espectadores de cine, si bien la caída del número de espectadores masculinos ha sido un poco más intensa.

Téngase en cuenta que estos porcentajes parten de una cierta desigualdad en el reparto de la población por sexos, superando el número de mujeres ligeramente al de hombres (51,4\% frente a 48,6\%, respectivamente, según datos de 2013$)^{6}$.

En el caso de los diarios, los lectores eran un $62,7 \%$ en 2002 y $60,8 \%$ en 2014 , por $37,3 \%$ y $39,2 \%$, respectivamente, de lectoras. Este incremento porcentual de mujeres se explica por una menor caída del número de lectoras, de modo que si en 2002 la penetración respecto del total de cada sexo era del $26 \%$ de mujeres por $46,1 \%$ de hombres, en 2014 la proporción era de $24 \%$ y 39,2\%, respectivamente. Esta misma evolución es perceptible en el caso de suplementos y revistas. Aquí el incremento porcentual de las mujeres se explica por una menor caída del número de lectoras, afianzando el predominio femenino.

Respecto de los suplementos, se pasa de un porcentaje de lectoras de 50,4\%, con una penetración del $29,4 \%$, a un porcentaje de $56,7 \%$, con una penetración del $15,4 \%$; frente a un $49,6 \%$ de lectores en 2002 , con una penetración del $30,5 \%$, y de $43,3 \%$ en 2014 , con una penetración de $12,4 \%$.

Respecto de las revistas, se pasa de un porcentaje de lectoras del $55,7 \%$, con una penetración del $56,4 \%$, a un porcentaje del $57,1 \%$, con una penetración de 47,5\%; frente a un $44,3 \%$ de lectores en 2002 , con una penetración del $47,4 \%$, y de 42,9\% en 2014, con una penetración de $37,5 \%$.

La radio es, junto con internet, el único medio que ha experimentado un crecimiento global tanto en mujeres como en hombres, si bien el aumento más espectacular corresponde a internet ${ }^{7}$. También en ambos casos, si bien la mayoría corresponde a los hombres, se advierte una tendencia a la igualación por el mayor auge de oyentes e internautas mujeres.

De una parte, el porcentaje de oyentes femeninas en 2002 era del 45,9\%, con una penetración del 46,8\%, y del 47,5\% en 2014, con una penetración del 56,8\%; el porcentaje de oyentes masculinos en 2002 era del $54,1 \%$, con una penetración de 58,4\%, y del 52,5\% en 2014 , con una penetración del $66 \%$.

De otra parte, el porcentaje de internautas femeninas en 2008 era del 43\%, con una penetración del 22,9\%, y del 47,8\% en 2014, con una penetración del 51,8\%; el porcentaje de internautas masculinos en 2008 era del $57 \%$, con una penetración de 31,7\%, y del 59,5\% en 2014, con una penetración del $66 \%$.

De esta breve panorámica se infiere que en los medios en los que predomina o tiene un peso importante la función de entretenimiento, es mayoritaria la audiencia femenina o hay un reparto más equilibrado entre mujeres y hombres; así, cine, televisión, radio, suplementos, revistas e internet.

Una discriminación por temática ayudaría a precisar esta fórmula; en todo caso, es claro que hay revistas dirigidas a un público prioritariamente femenino y otras, a un público preferentemente masculino. De forma parecida, la programación y contenidos de radios, televisiones e internet abarca tanto entretenimiento como información; se dirigen en ocasiones a un público generalista, en otras, a segmentos de población.

\footnotetext{
${ }^{6}$ Marco General de los Medios en España 2014 - Distribución de la Población de 14 años o más por grupos de edad (p. 7 disponible en http://www.aimc.es/-Marco-General-html). Esta distribución por sexos se describe a través del índice de femineidad, esto es, la relación entre el número de mujeres y el número de hombres que conforman una población y que se expresa como el número de mujeres en un determinado año con relación a cada 100 hombres. Este índice en el año 2013 fue de $103,18 \%$ (Demografía Población e índice de feminidad, disponible en http://www.inmujer.gob.es/estadisticas/consulta.do?area=1).

${ }^{7}$ El crecimiento de internautas en España, visto en perspectiva, puede, en efecto, considerarse espectacular, pasando de $1 \%$ de internautas en los últimos 30 días en 1996 a 64,5\% en 2013; el incremento también se aprecia en el número de minutos diarios que pasan de 5,5 en 2000 a 90,5 en 2013 (Marco General de los Medios en España 2014, pp. 11 y 12). En los restantes medios disminuye el número de minutos diario en el mismo periodo, con la excepción del ligero incremento de radio y televisión; ahora bien, hay que considerar que internet ha alternado nuestra relación con los medios de comunicación, haciendo borrosas las fronteras tradicionales entre los distintos tipos de medios, de modo que en la actualidad es posible acceder a la versión digital de soportes tradicionales. Al respecto, vid. Internet en medio de los medios (2010) disponible en http://www.aimc.es/-Internet-enmedio-de-los-medios-.html.
} 
Por tanto, aun cuando la composición de la audiencia de determinados medios sea equilibrada en cuanto a participación de mujeres y hombres, cabe presuponer que unas y otros no compartan en la misma medida programas, contenidos y enfoques.

Al respecto es ilustrativo el caso de los diarios, especialmente si se compara con la lectura de suplementos.

El diario es un medio orientado prevalentemente a la información, debate y reflexión; y es el medio en el que se da una mayor desproporción entre audiencia femenina y masculina, con una ventaja evidente para los hombres. Ahora bien, son más las lectoras que los lectores de suplementos, pese a ser este un medio que acompaña a los diarios.

Al respecto, una mera constatación práctica revela numerosos ejemplos de magazines de prensa que, no siendo monográficos y encabezados por un título neutro, sin connotaciones de género, tienen un contenido dirigido exclusiva o mayoritariamente al público femenino (García de León, 2002: 86 nota 122).

El estudio Marco General de los Medios en España 2014 permite alguna precisión más respecto del tipo de medio de comunicación. Este estudio muestra una visión general de las audiencias de los diferentes medios españoles, de acuerdo con los datos del Estudio General de Medios (EGM), y su evolución en los últimos años. Conforme a la información suministrada, los diarios de información general tenían una penetración en 1997 del 32\%, pasando al 27,9\% en 2013; por su parte los diarios económicos pasaron del 0,4\% al 0,6\% en el mismo periodo, y los deportivos apenas experimentaron oscilaciones (10,8\% y 10,7\% en 1997 y 2013, respectivamente). En el año 2013, los diarios deportivos ocuparon los primeros puestos entre los diarios de pago con más audiencia (Marca, el primero y As, el tercero) ${ }^{8}$.

Por lo que se refiere a los suplementos, resulta significativo que medios que tienen como destinatarias inequívocas a las mujeres ocupen las primeras posiciones; así Mujer Hoy se encuentra en el segundo puesto de suplementos con más audiencia en $2013^{9}$.

Un panorama semejante se encuentra en las revistas semanales, con predominio de las revistas del corazón. Ha de advertirse que la información de la prensa del corazón tiene una connotación de frivolidad frente a la información considerada seria; esta percepción se mantiene en la actualidad si bien las fronteras entre los géneros periodísticos tienden hoy a difuminarse.

En las revistas quincenales y mensuales, la temática es más variada y las audiencias más repartidas. No obstante, es posible apreciar en un buen número de estas revistas un claro perfil de género; así por lo que se refiere a las publicaciones de moda (femenina) ${ }^{10}$.

\section{La audiencia de los medios de comunicación, género y contexto socioeconómico. Discriminación múltiple de la mujer}

Del estudio Marco General de los Medios en España 2014 claramente se desprende que el consumo de los medios de comunicación depende, en buena medida, del contexto socioeconómico que caracteriza la audiencia. Así, en el caso de los diarios, los lectores se encuentran, predominantemente, en las poblaciones de mayor tamaño y entre las clases sociales más altas, con un promedio de edad de 46,6 años (pp. 13-14).

Parece lógico suponer también que la elección de la temática de los medios y programas se relaciona con las actividades e intereses principales de su audiencia. Ello justifica el interés de considerar estas variables: empleabilidad y poder económico, formación, y uso del tiempo.

En 2013 la tasa de actividad de las mujeres era del $53,29 \%$, con una tasa de empleo del $38,89 \%$ y de paro del $27,02 \%$; en el caso de los hombres los porcentajes eran del 66,19\%,49,12\% y 25,79\%, respectivamente. En los años precedentes, las diferencias eran más acusadas; así, sin perjuicio de las oscilaciones derivadas de la coyuntura económica, se observa un incremento paulatino de la tasa de actividad de las mujeres que en 1996 era del $51,47 \%{ }^{11}$.

Más allá de la situación laboral, interesa también el poder económico y de decisión. Respecto del primero, las mujeres perciben salarios inferiores a los de los hombres ${ }^{12}$. Las mujeres reciben de media el 76,07\% de salario respecto a salario de varones, según datos de 2012 en España; el porcentaje es del $84,86 \%$ si se computan trabajos a tiempo completo y del 90,54\% si se computan trabajos a tiempo parcial. Respecto, del segundo, si tomamos como referencia la presencia de mujeres en los Consejos de Administración de las grandes empresas, los datos son desalentadores. En 2004, el porcentaje de mujeres era del 2,58\%, en 2012, del 13, 27\%; ahora bien, no se trata de una evolución lineal, pues se producen retrocesos, como en 2011, en el que el porcentaje cayó al 7,17\% ${ }^{13}$.

Si nos fijamos en la formación, encontramos mayoría de mujeres en los extremos, analfabetismo y enseñanza universitaria, con la excepción del doctorado. En efecto, en el año 2013, entre las personas analfabetas las mujeres alcanzaban el 68,7\%; en enseñanza

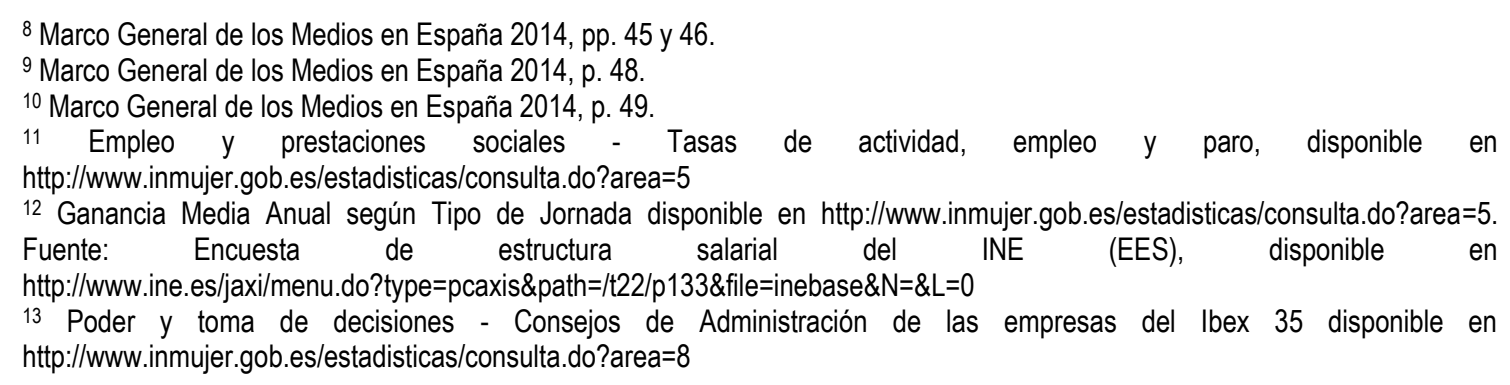


universitaria de primer y segundo ciclo, el 55,04\%; si bien en el tercer ciclo (doctorado), el porcentaje cae al 42,92\%. La evolución muestra una lenta mejora del nivel educativo de las mujeres desde el año 2000; en esta fecha, el porcentaje de mujeres entre las personas analfabetas era del 69,63\%; en educación superior (excepto doctorado) representaban el 48,68\%; en el doctorado, caían al 35,86\% ${ }^{14}$.

Este panorama se completa con el análisis del uso del tiempo de las mujeres y de los hombres ${ }^{15}$. Las categorías consideradas son las siguientes: cuidados personales, trabajo remunerado, estudios, hogar y familia, trabajo voluntario y reuniones, vida social y diversión, deportes y actividades al aire libre, aficiones e informática, medios de comunicación, trayectos y tiempo no especificado.

Según se deduce de las Encuestas de Empleo del Tiempo, a pesar de que los patrones en el empleo del tiempo de mujeres y hombres parecen estar aproximándose, existen diferencias significativas, tanto en el porcentaje de personas de cada sexo que dedica parte de su tiempo a las distintas actividades, como en el tiempo empleado en las mismas.

Las mayores diferencias se observan en el empleo del tiempo dedicado al cuidado del hogar y de la familia, a las aficiones y la informática, y al trabajo remunerado.

Las mujeres dedican menos tiempo que los hombres a todas las categorías de actividades, con la única excepción de aquellas que tienen que ver con el cuidado del hogar y de la familia. Esta categoría Hogar y familia se entiende constituida por actividades culinarias, mantenimiento del hogar, confección y cuidado de ropa, jardinería y cuidado de animales, construcción y reparaciones, compras y servicios, gestiones del hogar, cuidado de niños, ayudas a adultos miembros del hogar y a otras actividades para el hogar y la familia no especificadas.

De acuerdo con los datos de la última Encuesta de Empleo del Tiempo (2009-2010), el porcentaje de mujeres que empleaba tiempo en el cuidado del hogar y de la familia era del $91,9 \%$ y destinaban una media de 4 horas y 29 minutos diarios; el 74,7\% de los hombres destinaba 2 horas y 32 minutos. En el estudio 2002-2003, el porcentaje de mujeres era del 92,7\%, con una duración media de 4 horas y 45 minutos; frente al $70 \%$ de los hombres, con una duración media de 2 horas y 8 minutos.

Por su parte, de acuerdo con los datos de 2009-2010, el porcentaje de mujeres que destinaba tiempo a las aficiones y la informática era del $23,9 \%$, con una duración media diaria de 1 hora y 38 minutos; el porcentaje de varones era del 35,6\%, con un tiempo medio diario de 2 horas y 5 minutos. En los años 2002-2003, el porcentaje de mujeres era del 13\%, con una dedicación media diaria de 1 hora y 35 minutos; el porcentaje de hombres era el $23 \%$ y la duración media diaria, 1 hora y 59 minutos.

En cuanto al trabajo remunerado, el 28,2\% de las mujeres dedicaba, según el estudio de 2009-2010, una media diaria de 6 horas y 43 minutos; el 38,7\% de los varones empleaba 7 horas y 55 minutos. En 2002-2003, el porcentaje de mujeres era del 25,2\%, con una dedicación media diaria de 6 horas y 51 minutos; el porcentaje de hombres era el 43,3\%, con una dedicación media diaria de 8 horas y 22 minutos.

Por lo que se refiere a los medios de comunicación, en el estudio de 2009-2010, el 88,7\% de mujeres dedicaba a esta actividad una media diaria de 2 horas y 49 minutos; mientras que en los hombres el porcentaje era del 88\%, y una duración media de 3 horas y 6 minutos. En el estudio de 2002-2003, el porcentaje de mujeres era del $86 \%$ frente al $86,8 \%$ de hombres, y una duración de 2 horas y 28 minutos frente a 2 horas y 48 minutos, respectivamente.

Tras esta mirada global, merece la pena detenerse en el uso de internet por su incidencia en la inclusión y participación en la sociedad de la información (Sánchez Lería, 2011: 1389-1405). Precisamente, en este punto hace hincapié la Ley Orgánica 3/2007, de 22 de marzo, para la igualdad efectiva de mujeres y hombres, cuyo art. 28 (Sociedad de la Información) contemplaba el principio de igualdad de oportunidades entre mujeres y hombres en el diseño y ejecución de programas públicos de desarrollo de la Sociedad de la Información; la promoción de la plena incorporación de las mujeres en la Sociedad de la Información mediante el desarrollo de programas específicos, en especial, en materia de acceso y formación en tecnologías de la información y de las comunicaciones; la promoción de los contenidos creados por mujeres en el ámbito de la Sociedad de la Información; y finalmente, la garantía de que el lenguaje y contenidos fuesen no sexistas en los proyectos del ámbito de las tecnologías de la información y la comunicación sufragados total o parcialmente con dinero público.

A los datos ya vistos de dedicación de tiempo a actividades de informática y de audiencia de Internet, hay que añadir los derivados del análisis de visita de sitios de Internet (Visitantes únicos/Últimos 30 días) ${ }^{16}$ y sobre la brecha digital de género ${ }^{17}$.

Sobre la visita de sitios de Internet en España, se observa que las personas internautas se concentran en la clase media y alta, preferentemente, en la franja de edad entre los 25 y 44 años. Respecto al visionado de contenidos, se evidencia la inercia de los perfiles de género; así, en primer lugar se encuentra Youtube, en segundo lugar Marca, ocupando otro diario deportivo -As- el quinto lugar; en tercer lugar se encuentra El País; el cuarto, el sexto y el séptimo puesto, lo ocupan cadenas de televisión (Antena 3, RTVE y La Sexta, respectivamente).

14 Educación- Población de 16 y más años según nivel de formación alcanzado disponible en http://www.inmujer.gob.es/estadisticas/consulta.do?area=3

${ }^{15}$ Mujeres en Cifras. Boletín Estadístico - El tiempo de las mujeres y el tiempo de los hombres, $n^{0} 3$, marzo 2013, disponible en http://www.inmujer.gob.es/estadisticas/boletinEstadistico/docs/Boletin3Marzo2013.pdf

${ }^{16}$ Marco General de los Medios en España 2014 - Penetración \% 3a Ola Oct/Nov. 2013, pp. 61 y 63.

17 Mujeres y hombres en España Ciencia y tecnología, sociedad de la información (actualizado 8 marzo 2013); vid. punto 6.9.

Brecha digital de género, disponible

http://www.inmujer.gob.es/estadisticas/mujeresHombres/docs/2014/Mujeresyhombres2013.pdf 
La brecha digital de género se define como la diferencia del porcentaje de varones y el porcentaje de mujeres en el uso de TIC (ordenador, Internet, uso frecuente de Internet) expresada en puntos porcentuales. Estas variables proporcionan información del perfil de persona que los utiliza, de las diferencias de género en el uso, y del grado de desarrollo de las TIC en la sociedad.

Como señala el estudio, el acceso a la sociedad de la información forma parte del contenido de la ciudadanía en la actualidad. En este sentido, se ha elaborado una Agenda digital para Europa dirigida a fomentar una sociedad digital a través de la promoción del acceso a Internet y su utilización por todos los ciudadanos europeos, especialmente mediante actividades de capacitación para vivir y trabajar en la sociedad de la información, como la alfabetización digital.

Los datos demuestran que la brecha digital de género existe, si bien los valores en los tres indicadores analizados han descendido en los últimos años. En concreto, se reduce en el año 2012 respecto al año anterior en uso de ordenador y uso de Internet, excepto en el uso frecuente de Internet que aumenta un punto.

El valor más alto de la brecha entre mujeres y hombres corresponde al uso frecuente de Internet (6,7 puntos), seguido del uso de Internet (5,2 puntos) y del uso del ordenador (4,9 puntos).

Constatada la existencia de esta brecha digital de género, se exponen los factores que la explicarían; al margen de motivos técnicos y económicos (como la falta de infraestructuras), se destacan la falta de conocimientos de informática y habilidades necesarias para participar en la sociedad de la información, o la falta de interés en lo que la sociedad de la información puede ofrecer.

Este recorrido por la posición de la mujer en la sociedad actual muestra la facilidad con que la discriminación múltiple incide en la población femenina, al producirse la simultaneidad e interrelación entre dos o más formas de discriminación, con el consiguiente agravamiento de la situación de vulnerabilidad de la persona y mayores dificultades para el efectivo y pleno ejercicio de sus derechos (Serra Cristóbal, 2013: $15-43)^{18}$.

La situación es extensible a toda la Unión Europea. En este ámbito, la tasa de empleo de las mujeres continúa por debajo de la de los hombres (57,2\% frente al $71,6 \%$ en 2006), y siguen predominando en determinados sectores y en las profesiones menos valoradas y peor remuneradas (la diferencia de género en los salarios se sitúa en el $15 \%$ ). Son también las mujeres quienes habitualmente tienen trabajos a media jornada (31,4\% frente al 7,8 \% en 2007) y están infrarrepresentadas entre los directivos de las empresas y los agentes que adoptan las decisiones tanto en el espacio político como en el económico. Igualmente, destaca la mayor exposición de las mujeres a la pobreza, especialmente entre las que se encuentran en edad de jubilación (5 puntos porcentuales de diferencia de género), así como las madres solteras y las mujeres que pertenecen a grupos socialmente desfavorecidos, como las minorías étnicas y los inmigrantes ${ }^{19}$.

\section{Género, medición de audiencias y acceso a la información}

¿Son importantes las estadísticas para introducir la perspectiva de género en la investigación y análisis de la realidad social? La respuesta es afirmativa y así lo recoge la norma de referencia en la materia, la Ley Orgánica 3/2007, de 22 de marzo, para la igualdad efectiva de mujeres y hombres (art. 20 Adecuación de las estadísticas y estudios).

La primera cuestión que se plantea es si se observa sesgo de género en las encuestas relativas a medición de las audiencias de los medios de comunicación.

El estudio Marco General de los Medios en España 2014 advierte que la información referida a los medios de comunicación se completa con otros datos considerados relevantes; entre ellos, cifras sobre la población objeto de estudio del EGM, sacadas del Universo del Estudio General de Medios, así como datos acerca de los consumos, tanto de amas de casa como de individuos y, datos relativos a estilos de vida de los individuos, también obtenidos del EGM.

Al respecto se informa sobre la distribución de las amas de casa por situación laboral; distinguiendo la actividad del ama de casa en función de si trabaja 38,6\% o no trabaja 61,4\% (p 8). Igualmente constata la distribución de las amas de casa por grupos de edad; hasta 34 años, $13 \%$; entre 35 y 54 años, $42,8 \%$, y de más de 55 años, $44 \%$ (p. 9 ).

No se indica que parámetros se utilizan para definir al ama de casa; obviamente, no es la dedicación exclusiva a las tareas familiares y del hogar, pues contempla amas de casa que trabajan fuera del hogar familiar. En este sentido, se habla de la caída social del ama de casa y se pone esta figura como ejemplo de la fragmentación que experimentaría el rol de la mujer, obligada a adaptar su estatus tradicional a las nuevas realidades (García de León, 2001: 52 y 86-87).

Llama también la atención que no se considere la existencia de amos de casa. Ciertamente, el porcentaje de hombres que realizan tareas domésticas es, de media, inferior al de mujeres, y dedican a estos menesteres, también de media, menos tiempo diario. Ahora bien, minoritario o excepcional, hay un porcentaje de hombres dedicados exclusivamente o preferentemente a labores del hogar. $Y$ una estadística seria y objetiva no debería obviarlos sin más.

18 Vid. el Informe de la Agencia de los Derechos Fundamentales de la Unión Europea sobre discriminación múltiple [EU-MIDIS Data in Focus 5: "Discriminación múltiple" (Febrero 2011)].

${ }_{19}$ Manual para la perspectiva de género en las políticas de empleo, de inclusión social y de protección social, Dirección General de Empleo, Asuntos Sociales e Igualdad de Oportunidades, Unidad G1 Luxemburgo: Oficina de Publicaciones Oficiales de las Comunidades Europeas, 2008, p. 3. 
Esta realidad puede comprobarse al consultar el estudio de Personas Ocupadas en Empleo Doméstico y Limpieza Interior de Edificios, según el cual, en el año 2013 el porcentaje de mujeres empleadas domésticas era del 98,04\% del total, y el 86,59\% de otro personal de limpieza ${ }^{20}$

Resulta igualmente curiosa la distribución de categorías y productos a propósito del estudio sobre Consumo de individuos y amas de casa. Evolución del consumo de los individuos. Así, respecto del uso habitual de productos por parte de hombres y por parte de mujeres, resulta que el perfume solo es usado por mujeres (p. 66). Por otra parte, respecto del consumo semanal de las amas de casa, se consideran únicamente alimentación y productos del hogar (p. 67).

En conclusión, este estudio contempla a los hombres como individuos y específicamente como hombres; mientras que las mujeres entran en la categoría de individuos, de mujeres y de amas de casa. En realidad, ¿tiene sentido esta categoría de ama de casa si se reduce al análisis del consumo del hogar familiar?

La perpetuación de estereotipos tradicionales de género y la resistencia a la adaptación a los cambios experimentados en la sociedad, se aprecia en otros estudios sectoriales de audiencias. Cabe recordar que la ley para la igualdad efectiva de mujeres y hombres es de 2007.

Unos meses después de su aprobación, en junio de 2007, AIMC firmó un acuerdo marco con IAB Spain que sentó la base para la constitución de un grupo de trabajo (Grupo Digital) con el objetivo de definir las condiciones ideales para mejorar la medición de audiencias en medios interactivos. De manera expresa, pretendía continuar el avance de la mejora de la medición digital por las implicaciones que tiene en la evolución positiva del nuevo escenario de medios.

Como resultado de este mandato, las Juntas Directivas de IAB Spain y AIMC aprobaron en septiembre de 2008 la redacción final del Libro Blanco IAB-AIMC de Medición de Audiencias Digitales ${ }^{21}$. En él se contiene un listado de Buenas prácticas de la medición de audiencias digitales cuyo objetivo es ser un documento que sirva de referencia en la medición, lo suficientemente completo y flexible para perdurar en el tiempo (p.2).

Se reproducen a continuación los rasgos considerados para caracterizar la audiencia (5- Características de la Audiencia, p. 11). Las variables básicas del individuo contempladas son:

1. Sexo

2. Edad

3. Rol Familiar: Ama de Casa, Ama de Casa y Cabeza de Familia, Cabeza de Familia,

1. Otros individuos.

4. Estado Civil

5. Nivel de Instrucción

6. Situación Laboral: Trabaja / No Trabaja

7. Ocupación

8. Nacionalidad

Esta caracterización básica se completa con las variables del hogar:

1. Nivel de instrucción del Cabeza de Familia

2. Ocupación Laboral del Cabeza de Familia

3. Situación Laboral del Cabeza de Familia: Trabaja/ No Trabaja.

4. Clase Social (del hogar y del individuo): generada a través del Nivel de Estudios y Ocupación del sustentador principal de acuerdo al sistema de clasificación español.

5. Tamaño del Hogar.

6. Miembros que componen el hogar.

7. Relación existente entre los miembros que componen el hogar.

8. Edad de los miembros que componen el hogar.

9. Hábitat.

10. Provincia.

Obsérvese que, aparentemente, la mujer solo puede ser «ama de casa» o «ama de casa y cabeza de familia»; por tanto, únicamente podría ser considerada cabeza de familia en ausencia de un varón que ocupe ese puesto. En cambio, el hombre no es considerado en ningún caso como amo de casa. De nuevo, aparentemente, tampoco se contempla la posibilidad de que existan dos cabezas de familia; por ejemplo, una pareja en la que, con independencia de su sexo, ambos trabajan, comparten las tareas domésticas y toman las decisiones de forma conjunta.

Por tanto, la impresión inferida de esta caracterización es la de la consideración exclusiva de un modelo familiar jerarquizado, esto es, con una estructura piramidal ordenada por relaciones de subordinación y dependencia, y en la que la posición de cabeza de familia es ocupada de manera exclusiva por uno de sus miembros, con preferencia, un hombre. En otras palabras, un modelo de familia tradicional (Trigo García, 2011: 1435-1442).

Además de este - en mi opinión- marcado sesgo de género en el diseño de las encuestas, se echa en falta, siguiendo las previsiones de la normativa sobre igualdad, una consideración específica de la mujer. En efecto, no todas las estadísticas sobre audiencias de medios de

\footnotetext{
${ }^{20}$ Disponible en http://www.inmujer.gob.es/estadisticas/consulta.do?area=5

${ }^{21}$ Disponible en http://www.aimc.es/-Libro-Blanco-Digital-html
} 
comunicación consultadas desglosan los datos en función de la distribución mujer y hombre en cada una de las categorías examinadas. El caso extremo es el estudio Internet en medio de los medios 2010 que no discrimina en función del sexo.

Una recogida de datos más completa ofrecería más argumentos sobre la hipótesis aquí apuntada: la información sigue siendo cosa de hombres en cuanto protagonistas y en cuanto receptores; mientras que el público femenino tiene mayor presencia en formatos en los que prima el entretenimiento, si bien no siempre comparten contenidos, medios y programas con los hombres. En este sentido, sigue habiendo una clara diferencia de género en cuanto a los temas; por lo que se refiere a la audiencia femenina, siguen el perfil clásico de moda, belleza, cocina, etc.

Llama la atención que no existan análisis sobre categorías relativas a la cultura, con la excepción de la asistencia al cine, respecto la que, recuérdese, había un mayor número de espectadoras.

De nuevo, la atención a las manifestaciones culturales podría servir para reforzar la hipótesis de una diferencia de género por lo que respecta a información versus entretenimiento en la medida en que los datos relativos a empleo, poder de decisión, conciliación y ocio parecen indicar que las mujeres tienen una importante presencia en actividades culturales y de relación social, mientras que su papel es menor en el mercado laboral y en puestos directivos y políticos, lo que incidiría en un interés también menor por la información política, económica, científica y tecnológica; incluso, deportes ${ }^{22}$.

La conclusión sería que, como en otros ámbitos, también aquí se aprecia el que ha dado en llamarse «Efecto Penélope», esto es, una continua y contradictoria actividad del sistema social que tiene como objetivo lograr la igualdad social de género; coexisten prácticas sociales en un sentido y en el contrario que destruyen o atenúan los efectos de los primeros. De este modo la publicidad y en general los medios de comunicación destruyen (destejen) la igualdad formal de género que garantizaría la normativa vigente (García de León, 2002: 86).

Obsérvese que la menor audiencia femenina de medios de comunicación en los contenidos y categorías antes citados afecta a su capacidad para formar opiniones propias y tomar decisiones en ámbitos relevantes de su participación en la vida pública. Con las repercusiones consiguientes para el efectivo ejercicio de sus derechos como ciudadanas: la ciudadanía activa es una ciudadanía informada.

En general, la perpetuación de estereotipos de género desincentiva el acceso, tanto de mujeres como de hombres, a determinadas secciones, programas o medios de comunicación, lo que merma la diversidad y pluralidad cultural en una sociedad que adolece de rigidez en la configuración de los roles de género, todavía hoy marcados por los modelos tradicionales.

\section{Bibliografía}

Leonor Aguilar Ruiz, La publicidad sexista: nuevos instrumentos de regulación en derecho privado, El levantamiento del velo: Las mujeres en el derecho privado / dir. Ma paz García Rubio; Ma del Rosario Valpuesta Fernández; coord. Laura López de la Cruz; Marta Otero Crespo, tirant lo Blanch, Valencia, 2011, pp. 879-901

Inmaculada Berlanga Fernández, Comunicación audiovisual y mujer: evolución y nuevos formatos en las campañas publicitarias de violencia de género, Icono14, Vol. 9, № 1, 2011 (Ejemplar dedicado a: Mujeres y Tecnología), pp. 145-160

Estela Bernad Monferrer, Nuevos formatos publicitarios televisivos y perspectiva de género, Estudios sobre el mensaje periodístico, № Extra 18, 1 (Octubre), 2012, pp. 151-159

Mª Antonia García de León, Herederas y heridas. Sobres las élites profesionales femeninas, Cátedra, Madrid, 2002

Pilar López Díez, Los medios de comunicación y la publicidad como pilares fundamentales para lograr el respeto a los derechos humanos de las mujeres, Las mujeres en la Constitución europea: estudios multidisciplinares de género / coord. Ángela Figueruelo Burrieza, Salamanca, Ediciones Universidad Salamanca, 2005, pp. 67-90

Rafael López Lita, Estela Bernad Monferrer, Publicidad, medios de comunicación y segregación ocupacional de la mujer: perpetuación y superación de los estereotipos de género y sus consecuencias en el mercado de mano de obra, Revista del Ministerio de Trabajo e Inmigración, № 67, 2007 (Ejemplar dedicado a: Derecho social Internacional y Comunitario), pp. 213-226

María Macías, Políticas de igualdad de género y medios de comunicación, El Derecho en los medios de comunicación / coord. Alicia Duñaiturria Laguarda, Cristina Carretero González (dir.) Aranzadi, Cizur Menor (Navarra), 2013, pp. 207-226

María del Lirio Martín García, Mujeres, publicidad comercial y consumo: los códigos de conducta publicitarios, ¿una herramienta eficaz para la defensa de los intereses de mujer como consumidora, El levantamiento del velo: Las mujeres en el derecho privado / dir. Ma paz García Rubio; Ma del Rosario Valpuesta Fernández; coord. Laura López de la Cruz; Marta Otero Crespo, tirant lo Blanch, Valencia, 2011, pp. 903919

Isabel Menéndez Menéndez, Medios de comunicación, género e identidad, Sociología y género / coord. Capitolina Díaz Martínez, Sandra Dema Moreno, Tecnos, Madrid, 2013, pp. 253-270

María Salvador Martínez, Medios de comunicación e igualdad de mujeres y hombres, Género, constitución y estatutos de autonomía / coord. Teresa Freixes Sanjuán, Julia Sevilla Merino, Madrid, Instituto Nacional de Administración Pública, 2005, pp. 791-802

$M^{a}$ Carmen Núñez Zorrilla, La desigualdad por razón de género den los medios de comunicación. Especial referencia a su repercusión en el menor de edad, El levantamiento del velo: Las mujeres en el derecho privado / dir. Ma paz García Rubio; M ${ }^{a}$ del Rosario Valpuesta Fernández; coord. Laura López de la Cruz; Marta Otero Crespo, tirant lo Blanch, Valencia, 2011, pp. 921-955

Inés Pérez Marín, Autorregulación: análisis de las resoluciones del jurado de autocontrol de la publicidad discriminatoria de la mujer, El levantamiento del velo: Las mujeres en el derecho privado / dir. Mª paz García Rubio; Mª del Rosario Valpuesta Fernández; coord. Laura

22 Vid. Gender-Sensitive Indicators for Media, cit., p. 44; el estudio propugna la igualdad de género en las distintas categorías, secciones, áreas y temas de los medios de comunicación. 
López de la Cruz; Marta Otero Crespo, tirant lo Blanch, Valencia, 2011, pp. 983-115

Amalia Rodríguez González, Últimas aportaciones normativas sobre publicidad discriminatoria en el derecho internacional y europeo, El levantamiento del velo: Las mujeres en el derecho privado / dir. Ma paz García Rubio; Ma del Rosario Valpuesta Fernández; coord. Laura López de la Cruz; Marta Otero Crespo, tirant lo Blanch, Valencia, 2011, pp. 1017-1043

Reyes Sánchez Lería, Las mujeres y los servicios de la sociedad de la información: un diagnóstico, El levantamiento del velo: Las mujeres en el derecho privado / dir. Mª paz García Rubio; Ma del Rosario Valpuesta Fernández; coord. Laura López de la Cruz; Marta Otero Crespo, tirant lo Blanch, Valencia, 2011, pp. 1389-1405

Rosario Serra Cristóbal, La mujer como especial objeto de múltiples discriminaciones. La mujer multidiscriminada, Multidiscriminación en los ordenamientos jurídicos español y europeo, Serra Cristóbal, R. (coord.), Valencia, Tirant lo Blanch, 2013, pp. 15-43

Belén Trigo García, Autonomía de la mujer: la gestión de su persona y sus bienes, El levantamiento del velo: Las mujeres en el derecho privado / dir. Ma paz García Rubio; Ma del Rosario Valpuesta Fernández; coord. Laura López de la Cruz; Marta Otero Crespo, tirant lo Blanch, Valencia, 2011, pp. 1429-1471

\section{Cómo citar este artículo en bibliografías - How to cite this article in bibliographies / references:}

TRIGO-GARCIA, B. (2015): "Y papá lee el periódico. La perspectiva de género en la audiencia de los medios de comunicación y reflexiones de derecho antidiscriminatorio". En Revista de la Asociación Española de Investigación de la Comunicación, 2, pp. 32-40. 